\title{
Comment se forme un ministre colonial : l'initiation de Colbert, 1651-1664
}

\section{Philip P. Boucher}

Volume 37, numéro 3, décembre 1983

URI : https://id.erudit.org/iderudit/304181ar

DOI : https://doi.org/10.7202/304181ar

Aller au sommaire du numéro

Éditeur(s)

Institut d'histoire de l'Amérique française

ISSN

0035-2357 (imprimé)

1492-1383 (numérique)

Découvrir la revue

Citer cet article

Boucher, P. P. (1983). Comment se forme un ministre colonial : l'initiation de Colbert, 1651-1664. Revue d'histoire de l'Amérique française, 37(3), 431-452. https://doi.org/10.7202/304181ar d'utilisation que vous pouvez consulter en ligne.

https://apropos.erudit.org/fr/usagers/politique-dutilisation/ 


\title{
COMMENT SE FORME UN MINISTRE COLONIAL L'INITIATION DE COLBERT 1651-1664
}

\author{
PHILIP P. BOUCHER* \\ Département d' histoire \\ University of Alabama in Huntsville
}

Les historiens admettent volontiers que dans les affaires de l'État, Jean-Baptiste Colbert fut fidèle aux politiques de son illustre prédécesseur le cardinal Richelieu. Il l'affirme lui même en ces termes:

Je ne faisois que fournir la carrière dans laquelle le cardinal de Richelieu étoit rentré. De tout ce que le cardinal avoit entrepris pour la gloire du royaume, il N'y avoit rien de plus important que la navigation et le commerce. ${ }^{1}$

On est frappé, en effet, par le «mercantilisme» de Colbert, reflet fidèle de celui de son modèle, lorsqu'il s'agit de maintenir une marine militaire et marchande puissante et de privilégier les compagnies de commerce. Les historiens n'ont pas manqué de remarquer cette fidélité, ni d'ailleurs la pauvreté de documents sur les débuts de la carrière de Colbert: ils se sont par conséquent davantage penchés sur la période débutant en 1664, plus richement documentée. Colbert était-il vraiment préparé pour devenir le conseiller de Louis XIV en matière d'économie maritime? Question qui a reçu peu d'attention. En y regardant de plus près, on s'aperçoit que malgré sa foi au «mercantilisme» et son énergie bureaucratique, Colbert ne réussit pas à mettre sur pied une politique coloniale efficace. Pis encore, il ne disposa même pas des conseils d'une équipe d'experts en affaires coloniales pour prendre la relève de celle de Nicolas Fouquet, le détesté rival à qui il succéda. Le nouveau ministre n'était donc ni bien renseigné, ni bien conseillé comme administrateur des politiques coloniales. D'aussi sombres débuts ne furent donc pas sans amener une certaine confusion dans les premières initiatives de Colbert, sans parler de ses maladresses, lourdes de conséquences, en matière de politique coloniale.

* J'exprime ma gratitude à l'American Philosophical Society pour sa subvention couvrant une partie de la recherche en documentation de cet article. Je remercie également Al Hamscher, Harold Parker et ma collègue Carolyn White pour leur encouragement, leurs remarques et leurs judicieux conseils.

1 Testament politique de Jean-Baptiste Colbert (La Haye, 1693), 185. Cité par Claude Aboucaya, Les Intendants de la marine sous l'ancien régime (Gap, 1958), 34. Pour un excellent sommaire sur les idées politiques de Colbert, voir Charles Cole, Colbert and a Century of French Mercantilism (2 vols., N.Y., 1939), 1:357-474; C.J. Jaenen, «Le Colbertisme», Revue d' histoire de l'Amérique française, XVIII, 1 (juin 1964): 64-84; XVIII, 2 (septembre 1964): 252-266. 
Né à Reims, à l'intérieur du pays, c'est au poste d'intendant du cardinal Mazarin (1651) que Colbert s'initia aux affaires maritimes. Il était alors responsable des comptes, parmi lesquels figuraient les investissements de Mazarin dans de nombreuses compagnies de commerce ${ }^{2}$. Devenu l'homme de confiance de Mazarin pendant dix ans, il put acquérir une connaissance approfondie des affaires de la marine marchande, une certaine familiarisation avec les questions d'échanges commerciaux avec l'étranger, mais peu d'expérience des affaires coloniales. Dès 1651, le Cardinal lui confia le mandat de démêler la confusion qui régnait dans les comptes de la Marine; ce qu'il réussit, semblet-il, grâce à l'aide experte du vénérable d'Infreville ${ }^{3}$. Plus tard, Colbert recommanda à Mazarin de créer des compagnies de commerce pour le Levant et l'Afrique du Nord ${ }^{4}$, faisant valoir que non seulement Son Éminence pourrait en récolter d'intéressants profits,

mais mesme contribuer à restablir le trafic et le commerce dans ce royaume par la protection que Votre Éminence donneroit à une compagnie qui pourroit estre establie pour cet effet... et à l'exemple de laquelle tous les sujets du roy feroient de nouveaux efforts... pour establir d'autres compagnies... le commerce estant la principale partie qui maintient un Estat dans l'ordre, la splendeur et l'abondance. ${ }^{5}$

Certaines archives indiquent qu'en 1653, le Cardinal - et peut être aussi Colbert - engagea des capitaux dans une compagnie faisant des affaires apparemment prometteuses, en Guyane. Les mérites de cette entreprise sont vantés dans quelques opuscules et un livre que son auteur dédie à Colbert ${ }^{6}$. Cette affaire fut un échec, mais Colbert n'en continua pas moins à réclamer à Mazarin, l'établissement d'un programme de renouveau commercial.

Il faut rétablir ou créer toutes les industries même de luxe, établir le système protecteur... restituer à la France le transport maritime de ses produits; développer les colonies et les rattacher commercialement à la France; supprimer tous les intermédiaires entre la France et l'Inde; développer la Marine militaire pour proteger la marine marchande. ${ }^{7}$

2 Pour la relation des débuts de la carrière de Colbert, voir les oeuvres de Pierre Clément et plus particulièrement Histoire de Colbert et de son administration (2 vols., Paris, 1892).

3 Lettres, instructions et mémoires de Colbert, éd. Pierre Clément (10 vols., Paris, 18611882), 1:152, 181 .

Ibid., 1:195-198. D'après Paul Masson, Histoire du commerce français dans le Levant au XVII ${ }^{e}$ siècle (Paris, 1896), 116, ces compagnies n'eurent aucun succès.

5 Lettres... de Colbert, éd. Clément, 1:195.

6 Paul Boyer, sieur de Petit-Puy, Véritable relation... (Paris, 1654), dédicace. L'histoire de cette intéressante compagnie est relatée dans Philip Boucher, «A Colonial Company at the Time of the Fronde: The Compagnie de la Terre ferme de l'Amérique ou France équinoxiale», Terrae Incognitae, XI (1979): 43-58.

7 Cité par J. Morini-Comby, Mercantilisme et protectionnisme (Paris, 1930), 45-46. 
Mazarin continua à s'intéresser aux compagnies de commerce tout au long des années 1650. Il envisagea bien de laisser les Caraïbes aux Anglais dans l'espoir d'amadouer Cromwell pour obtenir de lui une alliance anti-espagnole ${ }^{8}$. Mais il investit des capitaux «personnels» dans des entreprises pour le Levant ${ }^{9}$, La Guyane ${ }^{10}$, et Madagascar. Les livres de comptes tenus par Colbert révèlent que Mazarin engloutit une petite fortune dans l'aventure commerciale du duc de La Meilleraye à Madagascar $^{11}$. L'heure était peu propice à de tels risques car, à ce moment, les marchands étaient une proie facile pour les vaisseaux espagnols, anglais, hollandais et même français.

Après 1657, Mazarin et Colbert s'intéressèrent à la Mer du Nord; Anglais et Français allièrent leurs forces pour tenter la conquête de Dunkerque et de Gravelines. À partir de 1655, Colbert de Terron, cousin de Colbert et intendant de marine à La Rochelle, devint en quelque sorte l'agent de renseignements de ce dernier en matière d'affaires maritimes $^{12}$. Il faut relever, parmi les documents d'importance pour la carrière de Colbert, les rapports de Terron sur les négoces assez louches de Fouquet et ses exploits de corsaire sur l'Atlantique ${ }^{13}$. Les inquiétudes de Colbert s'amplifièrent à mesure que la rumeur publique faisait état des installations de Fouquet à Belle-Ile, sa nouvelle île-forteresse au large de la Bretagne ${ }^{14}$. Les archives sont muettes en ce qui concerne les rapports de Terron après 1659, ce qui laisse supposer que Colbert était sûr que les espions de Fouquet contrôlaient le courrier. Les soupçons concernant Belle-Île et l'aversion qu'éprouvait Colbert pour les méthodes de Fouquet dans l'administration des finances royales, finirent par empoisonner les relations entre ces deux anciens alliés.

Sur son lit de mort, en 1661, le cardinal Mazarin recommanda vivement Colbert à Louis XIV. Le roi nomma Fouquet, Hughes de Lionne et Michel Le Tellier au très important Conseil d'en Haut. Mais du même coup, il confia à Colbert le mandat de surveiller les comptes

\footnotetext{
8 Cardinal Mazarin, Lettres pendant son ministère, éd. Gustave d'Avenel, 9 vols. dans Collection de documents inédits sur l' histoire de France, $1^{\text {ère }}$ série (146 vols., Paris, 1872-1906), $6: 159$.

Bibliothèque nationale $(\mathrm{BN})$, Mélanges Colbert, 101: fol. 83v.

10 Archives nationales (AN), Section moderne, $\mathrm{F}^{12} 502$, fol. 11

11 Lettres de Colbert, éd. Clément, 1:527. En 1658 la Meilleraye demanda au cardinal d'augmenter ses investissements. Archives des Affaires étrangères (AAE), Mémoires et Documents, France, 905: fol. 98.

12 On trouvera des exemples dans AAE, Mémoires et Documents, France, 891: fol. 229v et 903: fol. 204; BN, Mélanges Colbert, 101: fol. 39.

13 BN, Mélanges Colbert, 101: fols. 290-292. Mazarin lui-même donnait son appui à des opérations corsaires entre 1652 et 1653 . Voir Lettres de Colbert, éd. Clément, 1:527; 2, partie ii, $412 ; 1: 211$. On se rappellera que les corsaires écumaient les mers dans les années 1650.

14 BN, Mélanges Colbert, 116 bis: fols, 907, 991. Colbert de Terron avait quelque expérience de l'espionnage de frondeurs en puissance comme le Cardinal de Retz et le duc de la Meilleraye. Pour la relation des débuts de la carrière de Terron, voir Jules Sottas, «Le Gouvernement de Brouage et La Rochelle sous Mazarin (1653-1661)», Revue de Saintonge et d'Aunis, XLI (1924-1926), XLII (1926-1927), XLII (1928).
} 
«byzantins» de Fouquet. Comme il relevait directement du roi, Colbert en profita pour accuser Fouquet de fraude et laisser planer sur lui des soupçons de trahison. On disait notamment que Fouquet accumulait à Belle-Île canons, munitions et importantes forces navales. Selon Terron, les explications données par le puissant surintendant à l'effet qu'il s'agissait de faire de l'île un entrepôt commercial, n'étaient qu'un «beau pretexte» pour mettre sur pied une puissante armée de mer ${ }^{15}$. En avril 1661, le roi forma le nouveau Conseil du Commerce dont il nomma Fouquet président et Colbert l'un des quatre membres; les trois autres étaient Pierre Clément, allié du surintendant, Étienne III d'Aligre, apparemment ami de Fouquet, et Denis Marin, parent de Colbert. Le Conseil rédigea sept volumes de mémoriaux portant sur les moyens aptes à stimuler une économie stagnante ${ }^{16}$. Ces documents allaient sans doute être plus tard précieux à Colbert. Mais, pour le moment, son attention était accaparée par sa conspiration contre Fouquet. Des échanges épistolaires entre Colbert et Terron révèlent combien ces derniers étaient embêtés par certaines demandes de renseignements adressées par Fouquet à Terron concernant des affaires de navigation ${ }^{17}$. Il y avait, bien entendu, des affaires plus pressantes à expédier.

Bien que la version populaire des événements accorde davantage d'importance aux relations de Fouquet avec la maîtresse du roi et à l'opulence de la fameuse fête de Vaux-le-Vicomte au cours de l'été de 1661, Louis XIV, de connivence avec Colbert, avait, dès le mois de mai de la même année, pris la décision de faire arrêter Fouquet ${ }^{18}$. Dans ses mémoires, le roi fait part de sa crainte de voir Fouquet devenir «le maître de ses états» ${ }^{19}$. On ne s'étonnera donc pas de l'allure secrète des échanges entre Colbert et Terron en avril et mai 1661, concernant le dossier des activités de Fouquet à Belle-Île ${ }^{20}$. Manifestement, le dit dossier devait servir à Colbert de pièce à conviction pour étayer ses doutes sur la trahison de Fouquet.

L'arrestation de Fouquet mit fin abruptement à son ambitieux programme de renouveau maritime et colonial. Quant à Colbert, déjà en butte à d'innombrables difficultés de parcours, il lui aurait fallu deux ans pour préparer de nouveaux projets. Colbert était convaincu, à bon

\footnotetext{
15 Cet écrit ainsi que d'autres lettres de Terron ont été publiés dans le récent livre d'Inès Murat, Colbert (Paris, 1980), 99

16 Mémoriaux du Conseil de 1661, éd. Jean de Boislisle (3 vols., Paris, 1905), 1:133.

17 BN, Mélanges Colbert, 104: fols. 450-450v, 487, 526.

18 Louis XIV, Mémoires, éd. Dreyss (2 vols., Paris, 1860), 2:525; Lettres de Colbert, éd. Clément, 2, partie ii: 35; Voir aussi une lettre de Louis XIV dans Archives de la Bastille, éd. François Ravaisson-Mollien (2 vols., Paris, 1866), 1:363.

19 Louis XIV, Mémoires, éd. Dreyss, 2:254. Voir aussi «Ce qu'on dit de Belle-Île», BN, Nouvelles Acquisitions françaises (NAF), Collection Baluze, 150: fol. 30.

${ }_{20}$ Voir les lettres citées par Inès Murat dans Colbert, 95-96; Voir aussi BN, Mélanges Colbert, 106: fol. 480. La publication de certains passages de ces lettres est intéressante, sous réserve du parti-pris de Murat pour Colbert. Murat n'a pas consulté les Mélanges Colbert ni d'autres documents d'archives publiques.
} 
droit, qu'on ne pouvait mettre sur pied un programme sérieux d'affaires maritimes sans un remaniement du système financier et la création d'une marine adéquate. Objectifs peu faciles à atteindre dans le contexte économique désastreux des années 1661-1662. Par exemple, il aurait fallu un tour de force pour trouver les capitaux nécessaires à l'acquisition de la Lorraine et de Dunkerque ${ }^{21}$.

La préparation du procès de Fouquet avait tellement drainé l'énergie de Colbert qu'il lui en restait peu à consacrer aux questions de commerce. Néanmoins, il sut tirer de précieux renseignements du dossier concernant les affaires maritimes qu'il avait préparé pour confondre Fouquet.

Il semble évident que Fouquet exerçait sur les officiers supérieurs de la Marine une influence importante, quoique surprenante, comme en témoigne le fameux document sur la «conspiration» rédigé par Fouquet pour se défendre d'une arrestation imminente ${ }^{22}$. Certains documents qui furent saisis par la suite indiquent clairement que le titre de vice-roi d'Amérique fut acheté par Fouquet pour le bénéfice du marquis de Feuquière ${ }^{23}$. Le surintendant affirma par ailleurs qu'il pouvait compter sur le vice-amiral Neuchèze (affecté sur l'Atlantique); et un mémoire adressé à Colbert révèle que c'est avec l'or de Fouquet que le viceamiral acheta ses titres ${ }^{24}$. D'autres noms sont cités dans le même document, notamment ceux du duc de La Meilleraye, lieutenant-gouverneur de la bouillante Bretagne et chef de file de la colonisation de Madagascar; de même le nom de Godefroi, comte d'Estrades, ambassadeur de carrière. Il appert également que la plupart des capitaines de la Marine, y compris le fameux Duquesne, et presque tous les intimes conseillers de l'Amiral Vendôme, étaient des «créatures» de Fouquet. On découvrit que Concarneau, Belle-île, Le Havre, l'île de Ré et le Mont SaintMichel étaient érigés en forteresses pour résister aux armées du roi.

L'échec de la sélecte Commission de Justice à lever des charges de trahison contre Fouquet peut s'expliquer par le pouvoir oppresseur qu'exerçait ce dernier sur la Marine de France. Pourtant, selon Charles de la Roncière et d'autres historiens, cet état de choses aurait dû faire l'objet d'une procédure exemplaire ${ }^{25}$. Il aurait fallu pour cela instituer d'énergiques enquêtes auprès des officiers supérieurs de la Marine, lesquels pour la plupart avaient des liens étroits avec Fouquet. S'il avait

\footnotetext{
${ }^{21}$ Correspondance administrative sous le règne de Louis XIV, éd. G.B. Depping (4 vols., Paris, 1850-1855), 3:10-11.

${ }_{22}$ Pierre Clément a publié une version de ce document dans: Histoire de la vie et l'administration de Colbert, précédé d'une étude historique sur Nicolas Fouquet (Paris, 1846), 41-47. ${ }^{23}$ La confession de Feuquières apparaît dans AAE, Mémoires et Documents, Amérique, 5: fol. 22 .

${ }_{24}$ Clément, Histoire de la vie de Colbert, 45: pour le document «Créatures de Foucquet dans la marine», voir BN, Mélanges Colbert, 107 bis: fol. 1157. 5:314-321.
} 
suivi cette voie, Colbert aurait été obligé d'effectuer une purge en hautlieu et de remplacer le personnel démis; il préféra une méthode plus conciliante. Par exemple, après que Neuchèze, confondu, se fut éclipsé, Colbert et Terron intercédèrent pour lui auprès du roi ${ }^{26}$. Après que Duquesne, lui aussi soupçonné de malversations, se fût retiré sans pour cela apaiser les doutes à son sujet, il reçut la mission d'aller exterminer les navires de Fouquet ${ }^{27}$. Notez que tout cela se passait dans les six mois suivant l'arrestation de Fouquet et avant même que Colbert eût complété le dossier d'où La Roncière tire ses conclusions ${ }^{28}$. Même si Colbert était alors en possession du document «conspiration", Neuchèze, d'Estrades et Duquesne étaient rentrés dans les rangs au moment du jugement, en 1664.

Colbert éprouva d'autres difficultés à préparer la revitalisation de l'empire colonial de France. D'abord, le manque d'informations sur l'état exact des colonies, à ce moment. Il avait en main, il est vrai, les mémoires et documents d'archives de Fouquet. Jacques Savary de Bruslons confirme en effet que Colbert les utilisa ${ }^{29}$. Jusqu'en 1663, plusieurs autres documents alimentèrent le dossier relatif aux intérêts maritimes de Fouquet ${ }^{30}$. Un autre problème était celui de recruter une équipe de conseillers et d'administrateurs experts en affaires coloniales. Après tout, le dernier avant-poste de Guyane avait disparu en 1657; les précieuses petites Antilles étaient aux mains d'intérêts privés et leur commerce monopolisé par les Hollandais; Madagascar était le fief du duc de La Meilleraye, soupçonné d'être un frondeur. Grâce aux Jésuites, à Monseigneur de Laval et à d'autres personnes reliées à l'obscure Compagnie du Saint-Sacrement, on avait une meilleure connaissance de la Nouvelle-France, mais Colbert sentait probablement déjà que ces gens pourraient être antipathiques à sa définition de l'Empire.

Avant même d'instituer un programme de renouveau colonial, il était essentiel de consolider et de rationaliser l'autorité de l'État en

\footnotetext{
26 BN, Mélanges Colbert, 106: fols, 313-314, 328-336, 348.

27 Ibid., 103: fols. 811-813; vol. 105: fol. 129v. Duquesne, un voisin de Fouquet, est cité comme une de ses «créatures»: Voir Archives de la Bastille, éd. Ravaisson-Mollien, 2:4. Abraham et Jacob Duquesne furent les associés de Christophe Fouquet de Chaslain, cousin du surintendant, dans une compagnie faisant affaire en Acadie, une région à laquelle Nicolas Fouquet s'intéressait. René Beaudry, "Quelques documents nouveaux sur Nicolas Denys», Revue d'histoire de l'Amérique française, IX, 1 (juin 1955): 15-20. Duquesne semble aussi avoir été très près de Colbert de Terron.

${ }_{28}$ Colbert exerça cependant une certaine prudence lorsqu'il se prévalut des services d'individus tels que le capitaine Nicolas Gargot, du chevalier Clerville, et de La Garde Bélin, agent de Fouquet à Amsterdam. En ce qui concerne Gargot, voir BN, Mélanges Colbert, 108: fol. 178, 190 bis: 847-848. Pour ce qui est de Clerville, Correspondance administrative sous le règne de Louis XIV, éd. Depping, 1: 646-650. Pour La Garde Belin, AN (Marine), B ${ }^{2}$, fol. 65; BN, Mélanges Colbert, 115: fol. 56; 115 bis: fols. 672, 992; 121 bis: fols. 924-924v.

29 BN, NAF, Collection Joly de Fleury, 2510: fol. 111. Baluze, le libraire de Colbert, a colligé les écrits de Fouquet, y compris les documents relatifs aux charges de trahison contre ce dernier. BN, NAF, Collection Baluze, v.149, 150.

BN, Mélanges Colbert, 106: fol. 131; 116: fols. 118-119.
} 
matière de commerce et de navigation. Avant d'être investi officiellement de ses pouvoirs, Colbert devait franchir l'autorité de Vendôme, le grand Maître de la Navigation. Ainsi, en 1662, Terron informe Colbert que la famille Vendôme s'oppose au départ des navires du roi pour Terre-Neuve. «Je ne sais pas d'où leur vient ces fantaisies là», commente alors Terron $^{31}$. La situation devenait encore plus confuse avec l'autorité indue qu' exerçaient Lionne, Le Tellier et Le Brienne sur certaines affaires maritimes. Par exemple, c'est le comte de Brienne qui autorisa la nomination de Bertrand d'Ogeron comme gouverneur de La Tortue en $1662^{32}$. Le comte d'Estrades, à ce moment vice-roi provisoire d'Amérique, protesta avec véhémence auprès de Colbert de cette usurpation de pouvoirs ${ }^{33}$; c'était la première fois, semble-t-il, que Colbert entendait parler du gouverneur de Saint-Domingue, plus tard de célèbre mémoire. Ce n'est qu'en 1664 que Colbert commença à mettre de l'ordre dans toute cette confusion bureaucratique.

Colbert eut son lot de problèmes personnels dans la décade de 1660 à 1670. En premier lieu, peu de gens parmi les haut gradés du gouvernement avaient quelque expérience des affaires coloniales, exception faite d'Aligre, ancien ami de Fouquet, qui avait exercé ses activités au sein de plusieurs compagnies de commerce et siégé au Conseil de Commerce en 1661 en même temps que Colbert. On ne trouve que peu d'échanges épistolaires entre ces deux hommes, probablement à cause du fait qu'ils habitaient tous deux Paris; mais on sait que d'Aligre collabora avec Colbert dans la mise en route des compagnies pour les Indes orientales et occidentales, et sur d'autres questions d'ordre commercial $^{34}$. Les autres conseillers de Fouquet restèrent loyaux au nouveau ministre, notamment le chevronné Pierre Chanut qui travailla pour Fouquet à titre de coordonnateur des affaires coloniales, poste précédemment occupé par Fouquet père sous le règne de Richelieu. Avant la disgrâce de Fouquet, Chanut et Colbert ne se vouaient pas des sentiments très amicaux: Chanut traita un jour Colbert de «mauvais homme». Après l'arrestation, il écrivit au nouveau ministre une lettre très digne dans laquelle il réaffirmait son amitié pour le surintendant

31 BN, Mélanges Colbert, 108: fol. 87. Dès 1651, Colbert se plaignit de l'autorité qu'exerçait Vendôme sur la Marine. Voir P.A. Cheruel, Mémoires sur la vie publique et privée de Fouquet (2 vols., Paris, 1862), 2:315.

32. Moreau de Saint-Méry, Loi et constitutions des colonies françaises de l'Amérique sous le vent de 1550 à 1758 (6 vols., Paris, 1785-1790), 1:87-88.

33 BN, Mélanges Colbert, 111: fol. 481.

34 P. Du Tertre, Histoire générale des isles Antilles... (4 vols., Paris, 1667-1671), 3:30; AAE, Mémoires et Documents, France, 916. D'Aligre siégea comme directeur de la Compagnie des îles d'Amérique (1635-1648) et président du Conseil de la Marine, sous Anne d'Autriche et Vendôme. BN, NAF, Collection Joly de Fleury, 2510: fol. 111. Après l'arrestation de Fouquet le roi nomma d'Aligre au Conseil de Finance aux côtés de Colbert, du maréchal Villeroy et d'un autre membre. 
déchu $^{35}$. Il ne semble pas exister d'autres documents concernant les relations entre ces deux hommes.

Certains historiens contemporains interprètent la chute de Fouquet comme l'épilogue d'une guerre de «clans» ou de «factions». On constate, en effet, que les deux personnages s'étaient forgé un réseau d' «alliés» assez élaboré; toutefois, Fouquet était en meilleure posture pour mettre à exécution un programme de renouveau colonial, à cause de ses liens avec la Bretagne et du fait qu'il s'était solidement infiltré dans les milieux de la navigation. Quant à Colbert, il devait exercer une grande prudence jusqu'à ce qu'il eût estimé l'importance de l'emprise de Fouquet sur la Marine. Entre 1661 et 1664, Colbert de Terron, en qui Colbert avait pleine confiance, et l'habile comte d'Estrades, furent les conseillers les plus influents de Colbert. Terron était très à l'aise pour discuter d'affaires concernant le Canada mais donna rarement d'avis concernant les autres colonies ${ }^{36}$. De son côté, d'Estrades, grâce à son poste diplomatique aux Pays-Bas, était bien placé pour obtenir des renseignements essentiels sur les activités des Hollandais aux Indes orientales et occidentales. Après avoir survécu au document «conspiration» de Fouquet ${ }^{37}$, le célèbre défenseur de Dunkerque (1652) vit grandir sa renommée pour avoir réussi à acheter Dunkerque des Anglais en $1662^{38}$. Il reçut plusieurs honneurs parmi lequels sa nomination comme vice-roi d'Amérique consacre son expérience présumée en affaires de navigation ${ }^{39}$.

En 1662, le roi et Colbert durent soudainement s'intéresser aux affaires du Canada lorsque le gouverneur de Trois-Rivières, Pierre Boucher, et Monseigneur de Laval débarquèrent pour venir solliciter l'aide de la France à la colonie en difficultét ${ }^{40}$. Les hésitations et incertitudes de Colbert à la suite de cette démarche montrent combien il était peu

35 BN, Mélanges Colbert, 103: fols, 178, 497. Entre 1662 et 1664 , le Conseil de Commerce, semble-t-il, fonctionna de manière non officielle, en l'absence de Fouquet et de Chanut. Colbert, d'Aligre, Marin et probablement Alexandre de Sève se réunirent pour discuter de questions mercantiles. Voir, par exemple, un édit cité par Du Tertre, Histoire générale, 3:30.

36 BN, Mélanges Colbert, 108: fol. 87; 118 bis: fol. 606v; 119: fols. $41,180 \mathrm{v} ; 119$ bis: fol. 732. Voir aussi Sottas, «Le Gouvernement de Brouage», Revue de Saintonge et d'Aunis, XLIII (1928): 41-80.

37 Pour un témoignage de l'intérêt de d'Estrades pour le Canada et l'Acadie, et de ses liens possibles avec Fouquet, voir une lettre de Gargot apparaissant dans Gabriel Marcel, «Le Surintendant Fouquet, vice-roi d'Amérique», Revue de Géographie, 17 (1885): 138; BN, Mélanges Colbert, 108: fol. 178; Charles de La Morandière, Histoire de la pêche française de la morue dans l'Amérique Septentrionale (2 vols., Paris, 1962), 1:412.

38 Louis XIV, Mémoires, éd. Dreyss, 2:557; Lettres de Colbert, éd. Clément, 2, partie i: 63. Dans Archives de la Bastille, éd. Ravaisson-Mollien, 2:19, d'Estrades nie toute amitié avec Fouquet. 14,15

Les documents relatifs à sa nomination apparaissent dans: BN, Ms. fr., 10252: fols.

40 Les deux hommes reçurent un accueil favorable du roi. Voir Pierre Boucher, Histoire véritable et naturelle... (Paris, 1664), éd. G. Loffin (Montréal, 1852), préface. Boucher dédia son livre à Colbert. En 1662, l'aide au Canada était à son plus bas niveau parce qu'à cette époque, comme Lionne l'expliqua au gouverneur Du Bois d'Avaujour, la France était en proie à la famine et la maladie. AAE, Mémoires et Documents, France, 2135: fol. 11. 
au courant des affaires canadiennes. L'influence de Laval força le rappel de Du Bois d'Avaujour, un gouverneur au demeurant fort compétent. Ce qui surprend le plus c'est l'appui donné par d'Estrades et Terron à $\mathrm{Laval}^{41}$ qui réussit à imposer son propre candidat au poste de gouverneur.

Colbert devait pourtant sentir à ce moment l'hostilité grandissante des influents amis de Laval, membres de la Compagnie du St-Sacrement, à cause du rôle qu'il avait joué dans la condamnation de Fouquet et dans d'autres affaires. En 1662, le gouvernement était dans un tel brouillard à l'égard des affaires canadiennes, que Laval eût beau jeu pour exercer librement son influence. Colbert ne put rien d'autre qu'expédier, par le bateau du retour, un émissaire chargé de recueillir des renseignements plus objectifs sur la situation en Nouvelle-France. Ce n'est qu'en 1663 que Colbert prit connaissance de diverses notes à ce sujet; on remarque par exemple que Terron et d'Estrades virent sous un meilleur jour l'ex-gouverneur d'Avaujour, après quelques échanges avec $\mathrm{lui}^{43}$. Donc, s'il est vrai que les problèmes de la Nouvelle-France furent pour le nouveau ministre son baptême des affaires coloniales, la grâce de la confirmation lui fut d'autant plus réconfortante. Ce n'est qu'en 1665 que débarqua au Canada une équipe de fonctionnaires que Colbert tenait bien en main.

Le manque de personnel et de conseillers qualifiés constituait un problème encore plus cuisant en ce qui concerne les Antilles françaises. En accord avec Chanut, Colbert dût se rendre à l'évidence qu'il fallait remplacer les maîtres des îles; mais il manquait de candidats ${ }^{44}$. Tout vice-roi d'Amérique qu'il était, d'Estrades dut admettre qu'il ne connaissait personne aux îles ${ }^{45}$. En décembre 1662, Colbert eut en main des renseignements plus précis sur la situation, lorsqu'il reçut un document relatif aux activités de Fouquet à la Martinique ${ }^{46}$. Il décida de nommer un délégué spécial aux colonies d'Amérique. Comme d'Estrades se plaignait de la difficulté d'intéresser un maréchal de France à

\footnotetext{
$41 \quad$ Le point de vue de d'Estrades est rapporté dans BN, Mélanges Colbert, 112 bis: fol. 573. Voir AN, (Marine), B ${ }^{2} 1$, fol. $162 \mathrm{v}$ pour le peu d'appréciation de Colbert pour d'Avaujour. AN, (Marine), $\mathrm{B}^{2} 1$, fol. $162 \mathrm{v}$.

43 BN, Mélanges Colbert, 118 bis: fols. 606v, 698. Dans une lettre datée de 1663, Colbert reconnaît les capacités de D'Avaujour mais le trouve «bizarre et peu pratique». Cité par Marcel Trudel, La Seigneurie des Cents Associés, tome 1, Les événements (Montréal, 1979): 323.

44 Dans les lettres adressées à d'Estrades en 1662 et 1663 , Colbert se plaint de Vaudroques, gouverneur de la Martinique. AN, (Marine), $\mathrm{B}^{2} 1$, fol. $110, \mathrm{~B}^{2} 2$, fol. 132. Le vice-roi partageait ses vues. BN, Mélanges Colbert, 115: fols. 167-167v; 116: fol. 490. Voir aussi un mémoire datant d'octobre 1662 demandant à Colbert d'éliminer les propriétaires privés. $\mathrm{AN}$, (Col.) $\mathrm{C}^{8} \mathrm{~B} 1$, pièce 9 .

45 Dans une lettre, Colbert demande l'avis du vice-roi. Voir AN (Marine), B ${ }^{2} 1$, fol. 111.

La réponse d'Estrades est dans BN, Mélanges Colbert, 111: fol. 428.
66 BN, Mélanges Colbert, 116: fols. 118-119; AAE, Mémoires et Documents, Amérique,
5: fol. 19. 5: fol. 19 .
} 
un tel poste ${ }^{47}$, il proposa son ami Alexandre de Prouville de Tracy ${ }^{48}$. Colbert avait fait récemment la connaissance de ce gentilhomme lorsque, cousu de dettes et fuyant Paris, Tracy était venu solliciter un emploi au gouvernement ${ }^{49}$. C'est le fait d'être disponible plutôt que toute autre qualité particulière qui fit que cet homme, déjà sexagénaire, fut choisi comme administrateur aux colonies. D'autre part, comme assistant de Tracy à titre de gouverneur de la Guadeloupe, Colbert nomma un sycophante et intrigant notoire dont les seules lettres de créance semblent avoir été sa collaboration antérieure avec Mazarin et sa disposition à voyager $^{50}$.

Vers la fin de 1662, Colbert reçut des avis plus substantiels sur la Guyane de la part d'hommes d'affaires qui mettaient sur pied une nouvelle compagnie de commerce pour cette colonie, présumée porte de l'Eldorado. Le responsable de la nouvelle compagnie, Le Febvre de $\mathrm{La}$ Barre, tenait lui-même ses renseignements de gens ayant échoué auparavant dans leur tentative de commerce avec la Guyane ${ }^{51}$. En fait, le dossier de La Barre lui-même était peu reluisant et sa nomination à la tête d'une telle entreprise montre la grande carence d'alors de gens qualifiés. Son incompétence comme intendant de province avait déjà été signalée à Mazarin en 1659 par Colbert qui avait réclamé sa démission ${ }^{52}$. Le cardinal était tombé d'accord mais, comme d'habitude, n'avait pas donné suite ${ }^{53}$. Il est possible que La Barre aît été protégé par Le Tellier, son chef d'alors ${ }^{54}$. Plus tard, entre 1661 et 1662 , La Barre essuya plusieurs rébellions au cours de son intendance dans le Bourbonnais. Lorsqu'en septembre 1662, il eut vent de son imminente destitution, il chercha la protection de Colbert ${ }^{55}$. Sa nomination à la tête de l'expédition pour la Guyane date de ce moment: on peut donc présumer qu'elle constituait un bon arrangement pour les deux hommes. Vers la fin de 1663, les préparatifs de l'expédition par La Barre et Tracy allaient bon train, sous l'oeil vigilant de Terron. Ce dernier, tout autant que Tracy, se plaignit de l'incompétence de La Barre. En réponse, Colbert tint ces propos:

\footnotetext{
$47 \quad$ BN, Mélanges Colbert, 118: fol. 189.

48 Ibid., 117 bis: fol. 931. On estimait le poste de d'Estrades en Hollande trop important pour aller en Amérique. Voir AN, (Col.), B1, fol. 64.

49 BN, Mélanges Colbert, 113: fol. 712; 110: fol. 147.

50 Ibid., 118: fol. 390. D’Estrades n'avait jamais entendu parler de Du Lion. Ibid., 117 bis: fol. 930

$51 \quad \mathrm{AN},(\mathrm{Col}),. \mathrm{C}^{8} \mathrm{~B} 1$, pièce 10; Du Tertre, Histoire générale, 3:4, 13.

52 Clément, Lettres de Colbert, 1:387. Un manuscrit décrit La Barre comme un «esprit souple accomodant avec les traitans dans son intendance qui par ce moyen a fort augmenté ses biens». Cité par Richard Bonney, Political Change in France under Richelieu and Mazarin, 16241661 (Oxford, 1978), 209.

53 Lettres de Colbert, éd. Clément, 1:388, 402, 405-406.

54 Même Le Tellier se sentit obligé d'inviter l'intendant à «changer ses manières». Voir la lettre citée par Douglas Baxter, Servants of the Sword: French Intendants of the Army, 1630-1670 (Urbana, 1976), 58. À cette époque La Barre entretenait aussi de bonnes relations avec Fouquet. BN, Ms. fr., Collection Baluze, 150: fol. 198.

55 BN, Mélanges Colbert, 111: fol. 529-530.
} 
Et ce que l'on en doit esperer de bon, c'est que devant envisager l'emploi qu'il a, comme sa dernière ressource, il y a lieu d'esperer qu'il s'y ménagera d'une manière, que si les affaires ne vont pas assez bien sous sa conduite qu'il seroit à désirer, au moins ne tourneront-elles point à cette pente facheuse qui les gateroit absolument. Mais si l'on s'apperceveroit qu'effectivement il travaillait à se faire un establissement particulier independemment de la Compagnie, il seroit aisé d'y remedier promptement cette espèce de folie. ${ }^{56}$

La carrière controversée de La Barre en Guyane, aux îles et au Canada fut, semble-t-il, tout juste à la limite du «désastre». Commentant le choix de La Barre à ce poste, Colbert déplora plus tard la difficulté de recruter des hommes de talent pour des postes aux colonies:

Mais la difficulté est de trouver un sujet propre pour cet emploi, estant certain que ceux qui pourroient bien remplir ce poste ne veulent pas se hazarder à un si long voyage... 57

Plus que pour toute autre colonie, on manquait de renseignements surtout sur Madagascar. Bien qu'il fut chargé d'administrer les fonds de Mazarin dans l'entreprise de la Meilleraye à Madagascar, Colbert déplora amèrement le fait d'être tenu dans l'ignorance des transactions financières et des plans de la compagnie ${ }^{58}$. Colbert et La Meilleraye s'entendaient assez mal; et plus tard, vers 1663-1664, le fils héritier de ce dernier, le duc Mazarini refusa de vendre sa concession de Madagascar $^{59}$. Étienne de Flacourt, l'un des rares hommes d'expérience en place et ex-gouverneur de l'île, auteur du premier livre important sur le pays, venait de mourir. Il semble que Colbert ait discuté de la situation de Madagascar avec le frère de Flacourt qui, plus tard, présenta les mémoires de Flacourt à la Compagnie des Indes orientales nouvellement formée ${ }^{60}$. On reçut également des fragments d'information de Nacquart, intendant à Dunkerque et confident de d'Estrades, et de La Garde Bélin agent de Colbert à Amsterdam ${ }^{61}$.

À l'été de 1663, Colbert s'empressa de monter une compagnie de commerce pour les Antilles et fonda la même année la Compagnie pour la Guyane. Elle ne servit toutefois que de tremplin à la beaucoup plus puissante Compagnie des Indes occidentales, fondée en 1664. La Compagnie de Guyane avait réussi à établir une colonie à Cayenne. À partir

$56 \quad \mathrm{AN},(\mathrm{Col}),. \mathrm{c}^{8} \mathrm{~B} 1$, pièce 13 .

57 Ibid., un mémoire datant de 1675 critique sévèrement le leadership de La Barre à Cayenne. An, (Col.) $\mathrm{c}^{10} \mathrm{~A} 1$. Ses activités aux îles et plus tard en Nouvelle-France ont généralement été critiquées.

${ }_{58}$ Colbert protesta avec véhémence. Voir BN, Mélanges Colbert, 101: fol. 166.

59 Colbert estimait La Meilleraye «un homme très difficile». Lettres de Colbert, éd. Clément, 1:295

60 Louis Pauliat, Madagascar sous Louis XIV (Paris, 1886), 116-117. Des documents sur Madagascar apparaissent dans AN, (Col.), $\mathrm{C}^{5} \mathrm{~A} 1$.

61 BN, Mélanges Colbert, 121 bis: fol. 466. 
de cette base, la nouvelle compagnie pourrait étendre ses activités sur toute l'étendue des Caraïbes et ainsi supplanter le monopole des Hollandais. La Barre, Terron et les associés de la Compagnie pour la Guyane devinrent titulaires de la nouvelle Compagnie. Son objectif était d'établir des échanges réciproques entre la France, l'Afrique orientale (traite des esclaves), La Guyane (bétail et autres denrées), et les Petites Antilles (produits de plantation). Pour quelque raison obscure, la NouvelleFrance figurait également dans l'édit de concession. La mission de Gaudais en Nouvelle-France et l'expédition en Guyane en 1663 ne semblent aucunement reliées ${ }^{62}$. Il n'est pas non plus évident qu'à l'aube de 1664 , Colbert eût sérieusement étudié la possibilité que le Canada devienne un pourvoyeur des Antilles. Aucun des mémoires dont nous faisons référence à l'annexe de ce texte ne fait état d'un lien économique qu'on aurait envisagé entre le Canada et les Antilles. La présence de la Nouvelle-France dans le réseau ne fut, semble-t-il, qu'une banale improvisation - vertement critiquée d'ailleurs - dictée par la ferme conviction de Colbert qu'une compagnie puissante pouvait réussir là où la fragile et maintenant dissoute Compagnie de Nouvelle-France (février 1663) avait échoué.

On tarda à constituer la Compagnie des Indes orientales parce que, à l'inverse de ce qui se passait en Amérique, peu de jalons étaient posés. Colbert semblait s'en remettre à l'avis de d'Estrades et de Nacquart, son associé, qui lui fournissaient des renseignements de première main sur les activités des Hollandais aux Indes orientales. D'Estrades faisait valoir que les deux compagnies françaises devaient récupérer les négociants des Pays-Bas désenchantés des services des compagnies hollandaises ${ }^{63}$. Il pria instamment Colbert de tenter de persuader le roi de recevoir ces marchands à Dunkerque ou au Louvre ${ }^{64}$. C'est d'Estrades lui-même qui recommanda François Caron, vieux routier du commerce hollandais qui devait plus tard jouer un rôle très discuté dans la Compagnie des Indes orientales ${ }^{65}$. Nacquart faisait pour la future compagnie une publicité sans relâche: il distribuait des copies de sa charte en Flandre, écrivait des tracts sur son potentiel et allait même jusqu'à Antwerp pour y recruter une clientèle de commerçants ${ }^{66}$. Finalement, Colbert s'en remit à son correspondant à Amsterdam, le terne La Garde Bélin, pourvoyeur de navires et auteur de mémoires, tâches qu'il accomplissait déjà sous Fouquet ${ }^{67}$.

\footnotetext{
62 AN, (Col.), B1, fol. 98, 98v. Dans un mémoire au roi en 1663, Colbert exaltait les mérites de la future Compagnie des Indes occidentales et la richesse qu'elle engendrerait. Lettres de Colbert, éd. Clément, 2, partie i, cclix, cclxix.

63 BN, Mélanges Colbert, 115: fol. 51.

64 Ibid., 121 bis: fol. $945 ; 123$ : fol. $171 ; 124$ : fol. $60 \mathrm{v}$.

65 Ibid., 121 bis: fols. $699,945$.

66 Ibid., 120: fol. 36v; 121 bis: fol. 466 .

67 AN, (Marine), B ${ }^{2} 1$, fol. 65; BN, Mélanges Colbert 115: fol. 56; 115 bis: fols, 672, 992; 121 bis: fols. 924-924v. La Garde Bélin finit par décevoir Colbert. Voir AN (Marine), B²5, fol. 73.
} 
Sur quel fondement sérieux Colbert se basa-t-il pour concevoir ses projets de 1663 et 1664 ? Certes, il épousait la tendance «mercantiliste» de l'époque, mais on la perçoit très peu dans les structures et les visées des compagnies qu'il mit sur pied. Entre 1661 et 1664, Colbert reçut plusieurs mémoires et en sollicita spécifiquement d'autres sur les affaires de commerce et les affaires coloniales; nous en donnons une liste assez complète en annexe. On peut y distinguer trois tendances particulières:

$1^{\circ}$ un accord sur la nécessité des compagnies de commerce mais un désaccord sur l'étendue de leurs privilèges;

$2^{\circ}$ un consensus sur le fait que renouveau commercial supposait action pour tenter de contrôler la suprématie des Hollandais sur les marchés de la France;

$3^{\circ}$ une importance dominante donnée à la Guyane et aux Antilles comme portes de l'empire atlantique.

On ne connaît pas le crédit réel de chacun de ces mémoires, même si les décisions finales de Colbert laissent entrevoir que certains furent mieux agréés que d'autres. Il les discuta probablement avec d'Estrades, Terron et d'autres ${ }^{68}$. Mais les échanges verbaux ne sont hélas! pas disponibles à l'historien qui veut reconstituer le cheminement de l'homme politique.

Les auteurs des mémoires s'accordaient pour reconnaître le déclin de la navigation française et recommander l'intervention du roi pour la sortir de l'ornière. Ils déploraient la suprématie qu'exerçaient sur les mers les Anglais et les Hollandais. L'unanimité se faisait aussi autour de certaines propositions de solution: le renforcement de la marine de guerre, la restructuration de la marine marchande, le rappel des marins français en service outre-mer, la création de chambres de commerce et la reconstruction des colonies. Plusieurs auteurs encourageaient la création de compagnies privilégiées comme fer de lance à la renaissance de l'Empire. Vu la faiblesse financière de l'État à pouvoir établir des colonies, on préférait encourager les compagnies exerçant traditionnellement un monopole. On attribuait leurs premiers échecs à un manque de capital, de leadership et à des faits purement accidentels.

Il y eut tout de même des avis divergents à cet égard, comme en témoigne un mémoire du sieur Formont, influent banquier parisien et plus tard un des principaux actionnaires de la Compagnie du Nord (1669). Formont était d'avis que le roi devait écarter tous les étrangers du commerce de la France avec les pays d'outre-mer; plus particulièrement, ils devaient être bannis du lucratif commerce avec les Antilles françaises. Chiffres à l'appui, Formont montra les énormes profits que tiraient les Hollandais de ce commerce; il suggéra de leur donner un

${ }_{68}$ On fait état de ce genre de discussions dans BN, Mélanges Colbert, 119, bis: fol. 917v. 
délai d'un an pour liquider leurs actions, ce qui laisserait aux Français le temps de mettre sur pied leur propre réseau. À son avis, les négociants français devaient être invités à profiter de cette occasion et seraient sûrement heureux de le faire. Formont s'en prit violemment aux compagnies privilégiées et cita en exemple les outrecuidants avantages donnés à une compagnie parisienne (1657-1663) qui achetait de l'huile de baleine des Hollandais et la vendait en France au double du prix. Pas étonnant, s'exclamait-il, que les baleiniers français fussent attirés vers les ports hollandais ${ }^{69}$.

Colbert ne partagea pas l'avis de Formont quant aux responsabilités à donner aux commerçants français dans le commerce étranger. Ce groupe craignait bien trop que la constitution de colonies ne leur coûtât une petite fortune et ne rapportât que de maigres profits dans l'immédiat. Colbert resta fermement sur ses positions colonialistes traditionnelles à savoir que le pire obstacle à l'établissement de colonies était encore cette vision étroite et cupide des marchands français. $\mathrm{La}$ meilleure solution, semble-t-il, était encore le maintien des compagnies à monopole, étroitement contrôlées par l'État. Toutefois au début des années 1660, on s'interrogeait sérieusement sur l'envergure et le mode d'organisation de ces compagnies.

Colbert épousait les vues de Richelieu concernant les sociétés de commerce géantes. Les deux hommes commettaient cependant la même erreur d'attribuer le succès des compagnies hollandaises au fait de posséder un réseau commercial très étendu. Pourtant les sociétés nationales dont Richelieu avait planifié les structures n'avaient jamais finalement vu le jour. D'autre part, les petites compagnies à charte s'identifiaient à un ou deux ports et demeuraient trop faibles financièrement dans une perspective de commerce colonial. Cette perception empêcha l'aboutissement en 1663 d'une proposition de former une compagnie pour les Antilles dont le siège aurait été à Dunkerque. Colbert rejeta également la requête de marchands de Tours, d'Angers et de La Rochelle à l'effet de former une compagnie pour les Indes orientales dont le siège aurait été à Belle-Île ${ }^{70}$. On pourrait arguer que Colbert donna tout de même son appui à la Compagnie de Guyane (1663), petite compagnie de vingt associés et au capital de deux cent mille livres. Toutefois, La Barre soutenait que la colonie de Cayenne n'était qu'un tremplin à partir duquel la France pouvait s'attaquer au monopole hollandais dans les Caraibes et former une compagnie plus importante. Dans un deuxième temps, on pourrait dépêcher annuellement une dizaine de vaisseaux français aux îles ${ }^{71}$.

\footnotetext{
$69 \quad$ Annexe no 9.

70 Annexe nos 16, 19.

71 Annexe nos 13, 14.
} 
Colbert avait décidé que les grandes compagnies auraient la responsabilité de toutes les possessions françaises dans le secteur de leurs activités respectives et les secteurs constituant une «unité économique». C'est ainsi que la Compagnie des Indes occidentales avait sous son contrôle les postes de traite des esclaves d'Afrique, la Guyane, les Antilles et même la Nouvelle-France. Auparavant, vers 1650, d'autres compagnies comme la Guyane, avaient voulu créer de solides liens commerciaux avec la Martinique ${ }^{72}$. Tous les auteurs de mémoires de l'époque reliaient la Guyane aux Antilles, mais on était peu au courant des liens entre les îles et la Nouvelle-France. Que le Canada soit placé sous le contrôle de la Compagnie des Indes occidentales ne fut pas sans provoquer de vives protestations, notamment celles de l'intendant Jean Talon.

On doit insister sur le fait que c'est la Guyane qui représentait aux yeux de Colbert, le point de départ d'un nouvel empire colonial français. Cette région était préférée parce qu'on disposait de conseillers compétents et de recrues pour des expéditions éventuelles. Entre 1643 et 1657, quatre importantes expéditions ont atteint la «côte sauvage» de la Guyane. Elles essuyèrent toutes un magistral échec. Malgré tout, les auteurs de mémoires datant de l'époque de 1661 à 1664 s'accordent pour dire que la Guyane était le meilleur choix pour l'établissement d'une base coloniale. Ils attribuaient l'échec des expéditions antérieures, non pas à la rudesse du climat des Tropiques, mais plutot à l'inexpérience des colonisateurs et à une direction trop autocratique ${ }^{73}$. Après tout, disait-on, les Hollandais n'avaient-ils pas établi une colonie prospère à Cayenne après 1654, après que les Français y eurent euxmêmes échoué? La Guyane faisait naturellement bloc avec les îles parce qu'elle pouvait fournir bétail et provisions diverses utiles aux planteurs ${ }^{74}$. Outre le commerce, on croyait ferme aux chimères de l'Eldorado: $\mathrm{La}$ Barre lui même s'y laissa prendre comme en témoignent ses nombreux voyages de reconnaissance en 1665 .

Il faut établir une distinction d'ordre chronologique entre deux attitudes de la France à l'égard des Hollandais: l'une marquée d'hostilité après 1667, l'autre scellée par un pacte d'amitié en 1662 . Un mémoire, attribué à La Garde Belin, confirme cet état de choses. L'auteur recommande à la France de chasser les Hollandais de la Guyane

\footnotetext{
72 Project du dessein de la compagnie formée pour la terre ferme de l'Amérique, Photostat Americana, 2nd series, no. 101 ([Paris, 1653], Boston, 1940), 10. Discours servant d' instruction aux personnes qui désirent s'associer... dans la compagnie de la Terre ferme de l'Amérique méridionale (n.p.n.d. [Paris, 1657]), 32.

73 Abbé Biet, Voyage de la France équinoxiale (Paris, 1664), préface, 174-175; Pierre Pelleprat, Relation des missions... dans la Terre ferme de l'Amérique (Paris, 1655), 112; Le Febvre de La Barre, Description de la France équinoxiale (Paris, 1666), 1-5. Pour les expéditions de Guyane en 1655-1657, consulter Philip P. Boucher, «Shadows in the Past: France and Guiana 1655-1657», Proceedings of the French Colonial Historical Society, 6 (1981): 13-26.

74 Annexe nos 3, 8, 10.
} 
parce que, entre autres choses, ils ont fourni des armes aux Indiens. Toutefois, il propose que la France achète des vaisseaux et des provisions en Hollande et il montre, chiffres à l'appui, qu'il est préférable que les colons français s'approvisionnent d'esclaves auprès des Hollandais ${ }^{75}$. Ce n'était guère le genre de suggestions qu'appréciait Colbert, lui qui ne nourrissait pas les meilleurs sentiments à l'égard des Hollandais. Un autre mémoire datant de la même époque ne retint pas davantage son attention: on y recommandait le maintien des Hollandais à la Martinique et même de leur permettre d'y construire un fort ${ }^{76}$.

Les mémoires de La Barre trouvaient meilleure résonance avec les vues de Colbert. La future compagnie pour la Guyane se proposait d'éliminer les Hollandais des Caraïbes françaises. On devait restreindre le nombre des associés afin de sauvegarder une certaine confidentialité. La Barre rejeta d'emblée la candidature des marchands qui désiraient faire des profits rapides; en sorte que l'on chercha peu à rallier des associés ${ }^{77}$. La compagnie projetait d'installer à Cayenne une base prospère qui ne devait pas coûter plus de deux cent mille livres. Après quoi, on ferait connaître au public le succès de l'entreprise et on rallierait de nouveaux sociétaires. Le scénario évoluerait alors vers l'élimination du monopole hollandais sur le commerce des Caraïbes. On demanda au roi de fournir seulement deux navires; on le pria d'annuler les concessions antérieures consenties en Guyane et d'assouplir les règlements douaniers relatifs à l'importation de sucre et autres produits des îles ${ }^{78}$. On estimait qu'il ne faudrait à la compagnie que deux ans «pour ramener en france le Comerce des Isles et de La terre ferme», commerce évalué à six millions de livres.

Colbert sollicitait encore des mémoires sur les Caraỉbes alors même que la compagnie s'apprêtait secrètement à partir. L'auteur d'un mémoire suggérait d'accorder aux Hollandais un préavis d'un an avant de les expulser; il ajoutait cependant qu'ils ne pourraient être remplacés adéquatement que par une compagnie puissante ${ }^{79}$. Colbert compila tous les renseignements essentiels concernant les îles; il s'enquit par exemple de l'existence d'un conseil souverain à la Guadeloupe ${ }^{80}$. Il est intéressant de relever que certains correspondants n'avaient pas très bien réalisé la profondeur de sa phobie des Hollandais ${ }^{81}$. D'Estrades à La Haye, Nacquart à Dunkerque, et La Garde Bélin à Amsterdam désiraient raf-

\footnotetext{
75 Annexe no 3.

76 Annexe no 8. Cependant au cours des années 1662-1663, Colbert n'exprima pas d'animosité ouverte envers les Hollandais. Lettres de Colbert, éd. Clément, 2, partie ii: 412-415.

Annexe no 13. De toute manière, disait-il, la piètre performance des expéditions antérieures en Guyane faisait que peu de marchands étaient désireux de s'associer à la nouvelle compagnie.

Annexe no 14

Annexe no 18 .

Annexe nos 15,17

Annexe no 16.
} 
fermir le commerce français sans toutefois toucher au traité d'amitié de 1662. Nacquart proposait que sa compagnie, dont le siège serait à Dunkerque, achetât des navires à Amsterdam et que les produits des îles y fussent expédiés pour être ensuite réexportés ailleurs. Il pria même le roi d'exercer son influence auprès de la Compagnie hollandaise des Indes occidentales afin que sa propre compagnie puisse se procurer du bétail à Curaçao! Même s'il souhaitait ouvertement la destruction du monopole hollandais aux Caraïbes, il voulait que cela se fit sans préjudice pour les «alliés du roi [les Hollandais]» ${ }^{82}$. En 1664, après le départ de La Barre et de Tracy pour la Guyane, d'Estrades s'enquit de la possibilité pour certains entrepreneurs zélandais de fournir des esclaves aux Antilles françaises; la même requête fut réitérée l'année suivante ${ }^{83}$. L'ambiance du début de 1667 fut plus propice à ce genre de négoce: Français et Hollandais étaient alors alliés contre les Anglais. Certains commerçants hollandais et zélandais réclamèrent des privilèges commerciaux avec les colonies françaises, quittes à payer une indemnité forfaitaire à la Compagnie française des Indes occidentales ${ }^{84}$. Inutile de dire que ce genre de proposition laissait Colbert peu enthousiaste: il avait choisi la voie de l'exclusion totale des Hollandais. C'est vers la fin de 1667 que cette prise de position se manifesta ouvertement et c'est à ce moment que sonna le glas de l'amitié franco-hollandaise.

Il ne faudrait pas porter un jugement trop sévère sur les politiques qui amenèrent la déconfiture de la Compagnie des Indes occidentales. Colbert avait bel et bien accepté la suggestion de La Barre de faire de la Guyane le point de départ d'une attaque contre les «intrus» hollandais: cela montre qu'il était d'accord sur une possibilité de symbiose entre la «côte sauvage» et les Antilles. Même les auteurs de mémoires qui s'intéressaient d'abord aux îles partageaient ce point de vue ${ }^{85}$. On se souviendra que vers 1663 et 1664, Colbert était peu informé de ce qui se passait aux Caraïbes. Comment aurait-il pu savoir que la Guyane était plutôt mal située pour commercer avec les îles; que la colonie de Cayenne était, en temps de guerre, à la merci de tous les ennemis venus; que la rudesse du climat tropical et les maladies qu'il engendrait rendaient difficile toute forme de prospérité? La Compagnie des Indes occidentales mit trop d'efforts et d'énergie en Guyane. Quant à l'hostilité implacable de Colbert pour les Hollandais, qui finalement amena la compagnie à sa perte, n'était-elle pas la pierre angulaire de son programme de renouveau maritime? Peut-être qu'une attitude plus conciliante eût été plus propice à la stabilité et la prospérité de la colonie; mais alors l'élément catalyseur de la reconstruction de la Marine n'aurait pas vu le jour.

\footnotetext{
82 Annexe no 10.

83 Correspondance administrative, éd. Depping, 3:348, 353.

$84 \mathrm{AN},(\mathrm{Col}),. \mathrm{F}^{2} \mathrm{~A} 10$, pièce 49.

85 Par exemple, voir Annexe no 8.
} 
Du côté de l'empire commercial oriental dont il rêvait, Colbert éprouva d'énormes difficultés à se rallier des conseillers d'expérience. Les chefs de file de la Compagnie de l'Orient étaient le duc de la Meilleraye et Fouquet, dont le procès à l'époque entretenait chez Colbert beaucoup d'animosité. Au début de la mise sur pied de la Compagnie des Indes orientales, Colbert eut maille à partir avec le duc Mazarini, fils, héritier de la Meilleraye, à propos du rachat de la concession de Madagascar. Étant donné que seule une poignée de Français pouvait se vanter de connaître les eaux orientales, Colbert décida de prendre conseil de personnes ayant des liens officiels ou officieux avec les Pays$\mathrm{Bas}^{86}$.

Au cours de 1663, Colbert commença à recevoir des offres de commerce pour l'Orient. Le projet des marchands de Tours, Angers et La Rochelle, mentionné plus haut, reçut peu d'attention à cause de ses faibles investissements et aussi les liens qui le rattachaient à Fouquet ${ }^{87}$. En revanche, le mémoire de l'opiniâtre La Garde Belin fut beaucoup mieux accueilli parce que, sans doute, son expérience au sein de la Compagnie hollandaise des Indes orientales avait fait impression sur le nouveau ministre. La Garde Belin proposait de monter une compagnie géante au capital de douze à treize millions de livres, souscrit par des sociétaires recrutés dans toute la France. À son avis, les administrateurs de la compagnie devaient être recrutés parmi les riches négociants et quelques conseillers d'état chevronnés; les officiers supérieurs et les nobles devaient être rigoureusement tenus à l'écart. Il proposa aussi l'établissement d'une colonie puissante à Madagascar, pour servir d'entrepôt. Il est certain que le mémoire de La Garde exerça une forte influence sur Colbert puisqu'on retrouve plusieurs de ses propositions dans la charte même de la compagnie ${ }^{88}$.

Dès février 1664, Colbert avait pris la décision de lancer la Compagnie des Indes orientales qui se proposait de faire de Madagascar le point tournant de la colonisation en Orient. Donc, même si les historiens établissent une différence entre l'empire commercial de l'Orient et l'empire colonial d'Amérique, il faut reconnaître que le succès des compagnies de commerce de 1664 était essentiellement axé sur le développement de Madagascar et de la Guyane en tant que colonies. Tous les conseillers de Colbert firent l'éloge du choix de Madagascar, ou peut-être à l'inverse, ce fut Colbert qui imposa l'idée à ses conseillers. Les travaux de l'académicien Charpentier en 1664 faisaient l'éloge des

\footnotetext{
${ }^{86}$ Parmi les renseignements reçus des Pays-Bas figurait une copie de la charte de la Compagnie hollandaise des Indes orientales. BN, Mélanges Colbert, 119 bis: 872-873.

Annexe no 19. On trouvera une version de ce mémoire dans Pauliat, Madagascar sous Louis XIV, 386-395. L.-P. May affirme, sans conviction, que ce mémoire influença Colbert pour la mise sur pied d'une compagnie pour les Indes orientales. Voir May, «Nicolas Fouquet et la politique coloniale de Louis XIV», Revue d'histoire des colonies, 33 (1940): 70-83.

88 Annexe no 20.
} 
«charmes fantastiques» de l'île ${ }^{89}$. Plus tard, Colbert reçut un accablant mémoire qui mettait à nu, point par point, tout l'illusoire du travail de Charpentier et le mettait en garde contre les éventuels déboires d'une colonisation de Madagascar. Colbert communiqua le mémoire au roi avec le maussade commentaire que cela «n'était pas conforme» à ce qu'on attendait de l'auteur ${ }^{90}$. À ce moment, Colbert avait aussi en main un mémoire très explicite où l'auteur, un observateur d'expérience, faisait valoir qu'il faudrait accorder beaucoup de privilèges pour intéresser d'éventuels émigrants à Madagascar. L'une des mesures proposées était de fournir aux colons des esclaves malgaches. L'auteur ne tenait pas compte du fait que les tentatives de colonisation antérieures avaient échoué justement à cause de mauvaises relations francomalgaches ${ }^{91}$.

D'Estrades et Nacquart eurent leur mot à dire lors de la formation de la Compagnie des Indes orientales. Ils tenaient absolument à ce que les marchands aient un contrôle sur l'administration. Colbert sembla accepter ce principe et en informa La Garde Bélin. Des Pays-Bas parvint une dépêche de d'Estrades disant que cette position ministérielle y avait suscité un très vif intérêt ${ }^{92}$. En fait, la charte de la compagnie était tout à fait conforme aux principales attentes des marchands: elle contenait la promesse du roi de ne pas effectuer de saisie des biens de la compagnie, même en cas de dette importante; les parts sociales individuelles étaient inviolables, même celles d'étrangers, en temps de guerre; les propriétaires de commerce actif devaient posséder les trois quarts des voix à la direction, les autres étant celles des officiers supérieurs du roi possédant une bonne expérience des affaires de navigation. Mais au fond, Colbert gardait un certain contrôle de la compagnie par personne interposée, comme par exemple, celle du banquier italien Bellinzani. Des syndics nommés par le roi devaient en principe céder la place à un conseil d'administration le ler décembre 1664; mais ils restèrent en place jusqu'au mois de mars suivant. Colbert nomma d'office le chef de la colonie de Madagascar et le directeur délégué des opérations commerciales aux Indes orientales. Les marchands réalisèrent vite le peu d'indépendance de la compagnie et peu s'y rallièrent finalement. En tout état de cause, les difficultés chroniques de l'entreprise de Madagascar finirent par lui mettre à dos l'opinion publique vers la fin de la décennie.

\section{$* *$}

${ }^{89}$ Ouvrir à n'importe quelle page le livre de F. Charpentier, Discours d' un fidèle sujet $d u$ roi (Paris, 1664).

${ }_{90}$ Annexe no 22.

91 Annexe no 23. Colbert et la Compagnie des Indes orientales reconnaissent la nécessité de bonnes relations à cet égard. Pauliat, Madagascar sous Louis XIV, 136-137, 141.

92 BN, Mélanges Colbert, 123: fol. 59v. 
Nous avons voulu, dans cet article, montrer les difficultés des premières démarches de Colbert, après son accession au pouvoir. Cela a débuté vers le milieu de 1662, lorsqu'il commença à s'impliquer dans les affaires coloniales: le problème fondamental était alors celui de recruter des conseillers et du personnel compétent en affaires coloniales. Ses conseillers de confiance comme Terron et d'Estrades ne savaient pas grand'chose des colonies, en particulier des Antilles et de Madagascar. D'autre part, Colbert devait en partie s'en remettre à des gens comme d'Estrades, Duquesne, La Garde Belin, qui avaient appartenu, dit-on, au clan de Fouquet. Il est certain que les indécisions de Colbert venaient, pour une large part, de son manque de connaissance des questions coloniales; c'est ainsi qu'il fut amené à partager la croyance courante que la Guyane et Madagascar représentaient la porte du succès respectivement à l'ouest et à l'est. De part et d'autre hélas! ce ne fut qu'un coûteux échec.

Tous ces tâtonnements restent empreints de la désagréable rudesse idéologique de Colbert. Par exemple, la virulence de son hostilité envers les Hollandais dépassait de loin les opinions beaucoup plus pondérées exprimées à cet égard par les auteurs de lettres et de mémoires, au point de rendre ridicule le traité d'amitié de 1662 . Son obsession exagérée de la puissance de la machine commerciale hollandaise fut, certes, un élément d'incitation important du renouveau colonial et commercial, mais elle augmenta aussi les risques de guerre et de batailles sur le plan commercial. La sévérité de ses jugements sur les échecs des expéditions coloniales antérieures l'amena à poser des gestes autocratiques auprès de la Compagnie de Madagascar, de la Compagnie de Nouvelle-France et auprès d'exploitants privés des petites Antilles ${ }^{93}$. Tous les organismes, impliqués dans des opérations mercantiles ou coloniales et ayant été reliés de quelque manière avec Fouquet, reçurent un accueil frigide. Certains financiers étaient assez mécontents des pressions exercées par Colbert pour souscrire aux compagnies de l'est et de l'ouest, mais il resta intraitable à leur égard.

Les historiens ont qualifié, à bon droit, de «révolution» la chute de Fouquet en 1661 et l'avènement d'une nouvelle relève politique. Malheureusement, la conduite administrative des affaires coloniales entre 1661 et 1664 peut tout juste être qualifiée de continuité dans l'incertitude. Il a fallu une bonne dizaine d'années pour sortir Colbert de l'ornière administrative. Mais à partir de ce moment, Colbert accorda beaucoup moins d'importance à la Guyane comme fer de lance de la stratégie coloniale dans l'Atlantique. Il réalisa vite l'importance des

\footnotetext{
93 Trudel, dans La Seigneurie des Cent Associés, 366-371, fait une analyse mordante des injustes accusations soulevées contre la Compagnie de Nouvelle-France. Encore plus injustes étaient les remarques désobligeantes du roi à l'égard des propriétaires privés des petites Antilles, lesquels se révélèrent en fait d'excellents colonisateurs. Voir l'édit royal dépossédant les propriétaires dans Du Tertre, Histoire générale, 3:29.
} 
liens économiques entre les îles et la Nouvelle-France, initiative combien plus prometteuse que la fameuse filière Guyane-Antilles, de surfaite renommée. Colbert ne se départit jamais complètement de sa politique d'exclusion des étrangers du commerce colonial, principe qui fut visiblement nuisible aux colonisateurs; tout de même, l'abolition en 1669 du monopole de la Compagnie des Indes occidentales eut un effet bénéfique sur le volume d'affaires des Français. Colbert manifesta son appui à De Baas, gouverneur général des Antilles, en dépit du fait que ce dernier dérogea systématiquement à la règle d'exclusion des étrangers. Enfin, en 1669, Colbert rationalisa l'administration de la Marine, ce qui en fit une excellente pièce sur l'échiquier de la colonisation. Voilà donc comment, après une initiation qui connut sa part de déboires, Colbert finit par mener à bien dans les années 1670 les destinées de la France d'outre-mer. Malheureusement, les années d'après vécurent le déchirement de la guerre et ne furent plus propices au développement rapide de l'Empire.

\section{ANNEXE}

\section{Mémoires 1660-1664}

1 «Advis de 1660 pour le commerce». BN, NAF, 2510: fol. 58 suite.

2 «Mémoire touchant les nouveaux établissements en Amérique» (Janvier 1661). AN (Col.), $\mathrm{C}^{8} \mathrm{~B} 1$, pièce 6.

3 «Mémoire contenant les avis et sentiments de différents capitaines de navires, voyageurs et autres sur les moyens de former des établissements à l'Amérique méridionale» (1661, mais probablement rédigé vers février 1660). AN (Col.), $\mathrm{C}^{8} \mathrm{~B} 1$, pièce 5.

4 «Projet pour le commerce par G. Audorin» (Mai 1661) BN, Ms. fr. 8022: fol. 154 suite.

5 «Mémoire pour le commerce par G. Audorin» (Mai 1661) BN, Ms. fr. 8022: fol. 14 suite.

6 «Projet pour une compagnie pour l'Orient» (1661) BN, Ms. fr. 8022: fol. 154 suite.

7 «Mémoire pour la marine» (July 1661) AAE, Mémoires et documents, France, 912: fol. 35 suite.

8 «Relation des isles Antilles de l'Amérique en l'état qu'elles étaient l'année 1660 ». (1661) AN (Col.) $\mathrm{F}^{3} 39$, pièce 1 . Le même que $\mathrm{AN}\left(\mathrm{Col}\right.$.), $\mathrm{C}^{8} \mathrm{~B} 1$, pièce 4. 
9 «Mémoire sur le commerce des îles par le sieur Formont» (1662). AN (Col.), $\mathrm{C}^{8} \mathrm{~B} 1$, pièce 8 .

10 «Mémoire sur les moyens de retirer les isles des mains des propriétaires», (Octobre 1662). AN (Col.), $C^{8} \mathrm{~B} 1$, pièce 9.

11 «Mémoire sur l'établissement d'une nouvelle compagnie pour Cayenne avec une description du pays... par le père de Desbordes Martin». (1662) $\mathrm{AN}$ (Col.), $\mathrm{C}^{14} 1$, fol. 188 suite.

12 «Mémoire pour l'établissement d'une nouvelle compagnie pour Cayenne avec une description du Pais et de l'état ou sy trouve les Hollandois» (1662). AN (Col.), $\mathrm{F}^{3} 21$, fol. 43 suite.

13 «Mémoire sur les projets de la compagnie qui se forme pour le Cap du Nord de Guiane» [Le Febvre de La Barre] (1662), AN (Col.), $\mathrm{C}^{14} 1$, fol. 85 suite.

14 «Mémoire pour la compagnie de l'Amérique en France équinoctiale, des choses qu'elle estime devoir lui être accordée pour exciter les particuliers à y entrer» [Le Febvre de La Barre] (fin 1662 ou début 1663). AN (Col.), $\mathrm{C}^{14} 1$, fol. 87 suite.

15 «La Réponse à une demande de M. Colbert» (1663) [Mémoire d'Estrades]. AN (Col.), $\mathrm{C}^{8} \mathrm{~A} 1$, fol. 1 suite.

16 «Proposition au Roy d'une nouvelle compagnie à establir pour le commerce des isles françaises de l'Amérique» (1663). $\mathrm{AN}\left(\mathrm{Col}\right.$.), $\mathrm{C}^{8} \mathrm{~B} 1$, pièce 10.

17 «Réponse au mémoire de Monsieur Colbert» (1663). AN (Col.), $\mathrm{C}^{10} \mathrm{~B} 1$ (St. Christophe 1627-1667).

18 «Mémoire des moyens qu'il faudroit tenir pour empescher aux étrangers le négoce des isles de l'Amérique et de l'utilité qui en reviendront à la France» (1663). AN (Col.), $\mathrm{F}^{2} \mathrm{~B} 1$, fol. 3 suite.

19 «Projet de Compagnie des Indes Orientales» (1663). AN (Col.), C 2 2, fol. 9 suite.

20 «Mémoire pour establir la grande compagnie des Indes-Orientales». La Garde Belin (fin 1663, début 1664). AN (Col.), C²2, fol. 56 suite.

21 «Mémoire sur la compagnie des Indes [Orientales] (1664); auteur probable Charpentier, selon une note en marge. AN (Col.), $\mathrm{C}^{2} 2$, fol. 61 suite.

22 «Remarques de Sieur Gentillot sur les Indes» (1664). AN (Col.), C 22.

23 «Advis qu'un particulier prend la liberté à donner à Messieurs les directeurs de la compagnie des Indes Orientales, au sujet d'un établissement d'une colonie à Madagascar, et ce qu'il estime devoir être observé pour parvenir à la fin de leur desseins». Tiré de BN, Ms. fr., 6104 dans Cole: Colbert and a Century of French Mercantilism, 1:570-572. 